\title{
LA EDUCACIÓN A DISTANCIA EN LA FORMACIÓN INICIAL Y CONTINUA DE LA FACULTAD DE EDUCACIÓN DE LA PONTIFICIA UNIVERSIDAD CATÓLICA DEL PERÚ
}

\author{
(INITIAL AND IN SERVICE DISTANCE EDUCATION AT THE FACULTY OF EDUCATION OF THE \\ PONTIFICIA UNIVERSIDAD CATOLICA DEL PERÚ)
}

Rosa María Tafur Puente
Pontificia Universidad Católica del Perú (Perú)

\section{RESUMEN}

La educación a distancia constituye una modalidad alternativa válida en la formación profesional, tanto inicial como continua, de los estudiantes de la Facultad de Educación de la Pontificia Universidad Católica del Perú. Por su flexibilidad en tiempo, estrategias y estilos de aprendizaje, permite atender las demandas de capacitación y de perfeccionamiento de los docentes de distintos lugares del país, incorporando sectores de la población que no pueden acceder a la educación presencial por motivos diversos. Asimismo, posibilita que los estudiantes desarrollen autonomía en su aprendizaje, aprendiendo a su propio ritmo sin sufrir la presión de grupo; que potencien habilidades que les permitan discriminar información relevante, identificar fuentes de conocimiento y emplearlas, contrastar datos con la realidad, construir conocimiento y aplicarlo en forma adecuada. En suma, proporcionarles las herramientas que les posibilite continuar responsablemente su estudio permanente dentro de una cultura de aprendizaje, con miras a alcanzar el desarrollo humano.

El artículo muestra el proceso que ha habido en la Facultad de Educación en relación a la implantación de la educación a distancia dentro de su oferta académica, familiarizando al lector con los avances realizados en los últimos veinte años en esta modalidad de estudios.

Palabras clave: educación a distancia, desarrollo humano, modalidad mixta: presencial y a distancia, proyectos de desarrollo, diplomas de especialización. 


\begin{abstract}
Distance education constitutes a valid alternative delivery mode in initial and in service professional education for the students of the Faculty of Education of the Pontificia Universidad Católica del Perú. Because of its flexibility of time, strategies, and learning styles, it makes it possible to respond to the demands of pre-service and in-service development from teachers of different places of the country, and to involve sectors of the population who cannot enroll in face to face education for different reasons. At the same time, it allows students to develop autonomy in learning, to learn at their own pace without suffering from group pressure, to reinforce abilities that let them distinguish relevant information, to identify and use sources of information, to contrast information with reality, to construct knowledge and apply it adequately. In sum, to give the students the tools that let them continue their permanent study with responsibility, within a learning culture that tends to reach human development.
\end{abstract}

The article describes the process of implementation of distance education in the academic offer of the Faculty of Education, acquainting the reader with the latest development of this way of studying in the last twenty years.

Key words: distance education, human development, blended delivery mode: face to face and distance, development projects, specialization diplomas.

Una característica que distingue a la formación profesional de calidad es su posibilidad de adecuación a las demandas educativas del grupo humano al que forma, así como a los temas de nivel universal y nacional que inspiran a la educación en una determinada época.

El alcanzar el desarrollo humano entendido como "el proceso de expansión de las capacidades y derechos de las personas, dentro de un marco de igualdad de oportunidades, en el cual todas las personas pueden progresar en libertad" (PNUD, 2005, p. 5), y entendido también como una aspiración a alcanzar la libertad, el bienestar y la dignidad de todas las personas (UNESCO, 2002), engloba, de acuerdo al Programa de las Naciones Unidas para el Desarrollo (PNUD), tres rasgos que permiten medirlo: el gozo de una vida saludable, la adquisición de conocimientos y el acceso a los recursos necesarios para alcanzar un nivel digno de vida.

Dentro de estos rasgos, el acceso al conocimiento constituye un desafío y una responsabilidad que deben asumir las instituciones educativas de formación superior inicial y continua, más aún en países como el nuestro en los que la población es muy heterogénea y el acceso a la educación limitada. 
Para responder a la necesidad y a la tendencia cada vez más creciente de ampliar la cobertura educativa universalizando la educación permanente, la educación a distancia resulta una modalidad alternativa que permite cada vez mayor número de personas en el sistema educativo, sobre todo de aquellas que por razones diversas no pueden incorporarse a la educación presencial. La educación a distancia propone nuevas áreas de investigación y desarrollo para los alumnos, con una amplia perspectiva de atención a la gestión autónoma de su aprendizaje.

\section{NECESIDAD DE LA EDUCACIÓN A DISTANCIA ${ }^{1}$}

No existe un concepto único de educación a distancia. Las definiciones varían según el énfasis que imprimen los especialistas. Así por ejemplo:

Para Jara (1978) los sistemas de educación a distancia son modelos educativos destinados a satisfacer necesidades o demandas socioculturales que emergen del hombre o de una comunidad en proceso de desarrollo.

Perraton (1993) define a la educación a distancia como un proceso educativo en el cual el aprendizaje se realiza bajo la dirección de un especialista que se encuentra alejado del discente tanto en tiempo como en espacio.

García Aretio (1999) integra los conceptos vertidos por varios autores en base a rasgos reiterativos que encuentra en ellos, como son:

- Separación profesor alumno.

- Utilización de medios técnicos.

- Organización de apoyo-tutoría.

- Aprendizaje individual.

- Comunicación bidireccional.

- Enfoque tecnológico.

- Comunicación masiva.

- Procedimientos industriales.

Atendiendo a ellos, define la educación a distancia como un "sistema tecnológico de comunicación bidireccional que puede ser masivo y que constituye la interacción personal en el aula de formador y alumno como medio preferente de enseñanza por la acción sistemática y conjunta de diversos recursos didácticos y el apoyo de una organización y tutoría, que propician el aprendizaje independiente y flexible de los estudiantes" (García Aretio 1999, p.15). 
Según la definición de los autores citados, y nuestra propia concepción de educación a distancia podemos afirmar que ésta es una modalidad educativa que basada en la aplicación de tecnologías interactivas para lograr el aprendizaje, se desarrolla sin limitaciones de tiempo, lugar o edad de los alumnos.

Queda claro, entonces que no existe un consenso de qué se entiende por educación a distancia. Tanto el concepto como su operativización responden a la filosofía de las instituciones que la aplican, a la disponibilidad de los medios de comunicación que poseen, así como a las características propias de los usuarios. Sin embargo, a partir de las distintas experiencias de aplicación de la modalidad de educación a distancia, se cumplen ciertas variables que a decir de Capella (1999) le sirven de fundamento. Estas son: intencionalidad, tendencias, fases del sistema, investigación, tecnología y docencia-discencia.

\section{Intencionalidad}

El funcionamiento de la educación a distancia responde a las necesidades y tendencias existentes. Mientras que en los países desarrollados el énfasis se pone en la aplicación de alta tecnología educativa, en los países en vías de desarrollo la intencionalidad de su empleo es la democratización educativa. La necesidad de ampliar la cobertura educativa se observa en todos los niveles educativos pero es en la educación superior donde se da con mayor profundidad en los diferentes países en los que se aplica.

Gracias a la revolución científico- tecnológica, a la globalización de la economía y a la internacionalización de los servicios, en los distintos niveles educativos sobre todo en educación superior, existe un avance en la rapidez de las operaciones y en la intercomunicación entre diferentes sistemas y ámbitos espaciales, lo que favorece la acción educativa en esta modalidad.

Para García Aretio la educación a distancia es la alternativa educativa que responde adecuadamente a problemas como "al aumento en la demanda social de educación; al interés por el estudio de una población estudiantil dispersa geográficamente o alejada de instituciones educativas, así como al de capas marginales de la población que de otra forma no hubiesen podido acceder a la formación; a la conveniencia de reducción de la carestía de los sistemas presenciales de educación; a la necesidad de flexibilizar el sistema convencional de educación y a las dificultades de los alumnos para seguir estudios sin los requisitos de espacio, asistencia y tiempo" (García Aretio, 1999, p. 14). 
Esta modalidad alternativa en educación responde entonces a una característica y a la vez necesidad generalizada en nuestro siglo: la universalidad de la educación permanente. Universalidad que no se puede llevar a cabo exclusivamente con una enseñanza presencial escolarizada durante toda la vida de los usuarios. La población tan numerosa y heterogénea demanda atención de formación y cultura diversificadas; objetivos que se pueden cumplir con la educación a distancia.

\section{Tendencias}

Existen dos posturas u orientaciones en el diseño de la educación a distancia: algunos autores como García Llamas (1986) consideran que en la educación a distancia se debe procurar que los estudiantes se liberen por completo de los condicionamientos espacio-temporales para que logren mayor libertad de estudio, para ello es menester emplear los medios humanos y tecnológicos adecuados que favorezcan el aprendizaje.

Otros autores como López del Puerto (1999) consideran ventajoso combinar el sistema de educación presencial con el de educación a distancia. De esta forma se aprovecha la tecnología, se ahorra en costos, se estandarizan contenidos y se puede extender los servicios a más personas. Sin embargo, indica Escotet (1980), siguiendo esta orientación se corre el riesgo de limitar las innovaciones que ofrece la modalidad con el riesgo de reproducir la misma estructura de la institución educativa convencional.

\section{Investigación}

Para Capella, los estudios que se han realizado en los últimos 40 años sobre la problemática de la educación a distancia han coincidido en señalar que existen dos tipos de problemas que se presentan como una constante: por un lado los problemas académicos referidos a la carencia de una tutoría sistemática en la que el tutor realice comentarios a la labor del estudiante que le permita elevar su rendimiento; la baja calidad del material escrito, la escasa posibilidad de aplicar el aprendizaje basado en problemas y de aprendizajes cooperativos, entre otros. Por otro lado los problemas administrativos centrados fundamentalmente en el costo de servicios, en el difícil acceso a equipos de comunicación, en la carencia de centros de estudio cercanos a los lugares de residencia de los estudiantes, en la ubicación de las instituciones educativas que ofrecen la modalidad. Limitaciones del servicio de educación a distancia que deben ser tomados en cuenta para cubrir los requerimientos esenciales y optimizar el sistema. 
Los requerimientos antes mencionados están referidos a la necesidad de producir material instruccional que tenga en cuenta el vínculo profesor alumno; al empleo de métodos de simulación, a la experimentación de situaciones educativas, a la interactividad en el proceso, a la función facilitadora del tutor que imprime una relación orientadora al alumno, a la retroalimentación, a la evaluación del aprendizaje oportuno y adecuado, entre otros.

\section{Tecnología}

Los mayoría de los sistemas de educación superior a distancia en América Latina se desarrollan siguiendo un mismo esquema: el empleo de material autoinstructivo de preferencia escrito, un sistema tutorial presencial, telefónico, postal y por correo electrónico. El esquema se ha visto enriquecido en los últimos años por la incorporación de la tecnología. Las innovaciones han ocurrido fundamentalmente tanto en el material educativo como en la labor tutorial. El material educativo impreso se ha complementado, si no reemplazado por el material colocado "en línea" en las plataformas virtuales. La labor tutorial se ha enriquecido mediante el correo electrónico, el chat y el foro.

Estas innovaciones han contribuido a que la educación a distancia pase rápidamente de "un modelo de tratamiento de la información básica unidireccional y lineal a otro sustancial distinto en el que se concibe la información como esférica o poliédrica, esto es, dinámica, con múltiples puntos de acceso y enlace, construida por el usuario según sus necesidades" (Capella, 1999, p. 30).

No es necesario entonces la presencia sincrónica de los educadores y alumnos, ésta es reemplazada por el "aula virtual". Se ha modificado así el tiempo pero también el espacio de los actos; las interacciones ocurren en espacios movibles.

\section{Docencia/discencia}

La posibilidad de realizar actividades masivas con un equipo mínimo de docentes y con independencia del tiempo cronológico y del espacio en el que interactúa el alumno, característica de la educación a distancia, exige y a la vez facilita los recursos para la autoeducación en la medida que posibilita el estímulo de la gestión autónoma de los discentes en la realización de su autoaprendizaje. Así, desde un diseño que potencie la autogestión en la resolución de problemas, la investigación, la observación personal, la elaboración creativa, la reflexión, el razonamiento crítico, acercará a los discentes a una autoeducación orientada. 
Estas consideraciones pueden sintetizarse, según Lamas en lo siguiente “(...) la finalidad última de un sistema a distancia es proveer a los alumnos de todos los elementos necesarios para facilitar su autoaprendizaje, todos los esfuerzos, todos los procesos están dirigidos hacia esto. Los materiales educativos están diseñados con la finalidad de que el hombre pueda aprender" (Lamas, 1999, p. 41).

Desde esta perspectiva, el rol presencial del docente se replantea: un equipo de personas especializadas en un área de producción de materiales educativos o en la conducción del proceso a distancia, como los tutores por ejemplo, se organizan para brindar al alumno la información adecuada que le facilite el aprendizaje. Estos nuevos roles implican diferentes relaciones interpersonales y por ende la necesidad de aprender a trabajar en equipo, de buscar actitudes conciliadoras y respuestas conjuntas que redunden en oportunidades de aprendizaje para los alumnos.

En suma, la educación a distancia debe ser planteada como un sistema que se preocupe de los sujetos que intervienen en él así como también de la interacción que ellos realizan con su medio ambiente. De esa manera no habrá soledad para el alumno en tanto se encuentre relacionado con su entorno natural, cultural y laboral.

Para el discente, el estudio a distancia significa la educación de su voluntad, lo que implica el desarrollo de la motivación intrínseca como motor que lo impulse en su aprendizaje y en la profundización de sus conocimientos, y el aprendizaje de datos, habilidades, actitudes, valores que tengan sentido y sean susceptibles de aplicación en el quehacer del alumno.

\section{LA EDUCACIÓN A DISTANCIA EN LA FACULTAD DE EDUCACIÓN DE LA PONTIFICIA UNIVERSIDAD CATÓLICA DEL PERÚ (PUCP)}

La Facultad de Educación fue creada en la convicción y el compromiso de "participar en el desarrollo de una sociedad justa y solidaria mediante la transformación y la humanización de las estructuras socio culturales" (Coloma, 1999, p. 2), y en coherencia con el espíritu de la Pontificia Universidad Católica del Perú. Espíritu nos hace reflexionar sobre la educación como un medio para alcanzar el desarrollo humano: única vía de freno a la explotación, a la violencia, a la pobreza, a la desigualdad, que atentan contra la sociedad.

Dentro de esta línea la Facultad de Educación, desde hace más de veinte años ha ido diversificando su atención en la formación de pre-gradoy en la formación continua, incorporando dentro de ellas a la educación a distancia como una alternativa en la 
atención de las necesidades emergentes de los estudiantes así como de los docentes y demás profesionales que apuestan por una formación responsable.

\section{Los inicios de la educación a distancia en la Facultad de Educación de la PUCP}

Para efectos del presente apartado el recorrido histórico de la Facultad de Educación en materia de educación a distancia se organiza en tres partes: la formación continua, la formación en pre-grado y los planes especiales de bachillerato y licenciatura.

\section{La formación continua a distancia}

La Facultad de Educación en concordancia con "el compromiso de la universidad de dar prioridad a la actualización, capacitación y especialización del magisterio nacional" (Tueros, 1999, p.160), en 1986 inició el primer proyecto de educación a distancia que ha servido como base de la creciente, competente y exitosa trayectoria de la que goza actualmente esta modalidad en nuestra Facultad. Esta experiencia empezó con el Proyecto de Profesionalización de Maestros de Zonas Andinas Rurales (Proyecto CRAM), gracias al apoyo académico y financiero de la Universidad de Mc Gill de Canadá y al de la Agencia Canadiense para el Desarrollo Internacional (ACDI). El Instituto Superior Pedagógico de Urubamba-Cusco, constituyó el centro de atención para otorgar el título profesional a los maestros de educación primaria de zonas rurales andinas. Fue una educación semi-presencial en la que se emplearon 12 módulos auto-instructivos que incluían material impreso, una guía de trabajo y un casete de audio.

En el año 1992 el proyecto Nuestros Niños y la Comunidad, extensión del proyecto inicial Nuestros Niños, Nuestro Futuro que empezó en 1982, brindó un curso a distancia con el objeto de atender a las docentes coordinadoras de programas no escolarizados de educación inicial pertenecientes a diversas ciudades del interior del país. Cabe anotar que este servicio se pudo realizar gracias al apoyo técnico de la Universidad de Winnipeg, Canadá y al financiamiento de ACDI. Se validó el material impreso consistente en 30 módulos de capacitación, 10 casetes de audio y 14 audiovisuales.

En el siguiente cuadro se muestra la evolución del proyecto Nuestros Niños, Nuestro Futuro entre 1982 y 1997: 
La Educación a Distancia en la Formación Inicial y Continua de la Facultad de Educación...

\begin{tabular}{|c|c|c|c|c|c|}
\hline Año & Denominación & Participantes & Localización & $\begin{array}{l}\text { Financiamiento } \\
\text { y apoyo técnico }\end{array}$ & Estrategia \\
\hline $\begin{array}{l}1982- \\
1985\end{array}$ & $\begin{array}{l}\text { Proyecto } \\
\text { Nuestros Niños, } \\
\text { Nuestro Futuro }\end{array}$ & $\begin{array}{l}\text { Perú } \\
\text { Bolivia } \\
\text { Ecuador }\end{array}$ & $\begin{array}{l}\text { Callao, } \\
\text { Villa El } \\
\text { Salvador } \\
\text { La Paz } \\
\text { Guayaquil }\end{array}$ & $\begin{array}{l}\text { AID, Washington, } \\
\text { High Scope } \\
\text { Michigan EE UU }\end{array}$ & $\begin{array}{l}\text { Educación } \\
\text { presencial }\end{array}$ \\
\hline $\begin{array}{l}1988- \\
1990\end{array}$ & $\begin{array}{l}\text { Proyecto Nuestros } \\
\text { Niños y la } \\
\text { Comunidad }\end{array}$ & $\begin{array}{l}\text { Dirección } \\
\text { Departamental } \\
\text { de Educación del } \\
\text { Callao }\end{array}$ & $\begin{array}{l}\text { Bellavista } \\
\text { Ventanilla }\end{array}$ & $\begin{array}{l}\text { ACDI Canadá } \\
\text { Universidad de } \\
\text { Winnipeg, Canadá }\end{array}$ & $\begin{array}{l}\text { Educación } \\
\text { presencial }\end{array}$ \\
\hline $\begin{array}{l}1990- \\
1991\end{array}$ & $\begin{array}{l}\text { Proyecto Nuestros } \\
\text { Niños y la } \\
\text { Comunidad }\end{array}$ & $\begin{array}{l}\text { Unidades } \\
\text { de Servicios } \\
\text { Educativos de } \\
\text { Lima }\end{array}$ & $\begin{array}{l}\text { Rimac } \\
\text { Chorrillos } \\
\text { Ate-Vitarte }\end{array}$ & $\begin{array}{l}\text { ACDI Canadá } \\
\text { Universidad de } \\
\text { Winnipeg, Canadá }\end{array}$ & $\begin{array}{l}\text { Educación } \\
\text { presencial }\end{array}$ \\
\hline $\begin{array}{l}1992- \\
1993\end{array}$ & $\begin{array}{l}\text { Proyecto Nuestros } \\
\text { Niños y la } \\
\text { Comunidad }\end{array}$ & $\begin{array}{l}\text { Direcciones } \\
\text { Departamentales }\end{array}$ & $\begin{array}{l}\text { Tacna } \\
\text { Chiclayo }\end{array}$ & $\begin{array}{l}\text { ACDI Canadá } \\
\text { Universidad de } \\
\text { Winnipeg, Canadá }\end{array}$ & $\begin{array}{l}\text { Educación a } \\
\text { distancia }\end{array}$ \\
\hline $\begin{array}{l}1994^{-} \\
1995\end{array}$ & $\begin{array}{l}\text { Proyecto Nuestros } \\
\text { Niños y la } \\
\text { Comunidad }\end{array}$ & 36 comunidades & $\begin{array}{l}\text { Ayacucho } \\
\text { Huancavelica }\end{array}$ & $\begin{array}{l}\text { Fondo General de } \\
\text { Contravalor } \\
\text { Perú-Canadá }\end{array}$ & $\begin{array}{l}\text { Educación a } \\
\text { distancia }\end{array}$ \\
\hline $\begin{array}{l}1994- \\
1997\end{array}$ & $\begin{array}{l}\text { Curso- Diploma } \\
\text { en Currículo y } \\
\text { Metodología en } \\
\text { Educación Inicial }\end{array}$ & $\begin{array}{l}\text { Lima y } \\
\text { distintos } \\
\text { departamentos del } \\
\text { país }\end{array}$ & $\begin{array}{l}\text { Piura, } \\
\text { Chiclayo } \\
\text { Trujillo (2) } \\
\text { Chimbote } \\
\text { Arequipa (2) } \\
\text { Cusco } \\
\text { Lima (9) } \\
\text { Ayacucho } \\
\text { Tacna }\end{array}$ & Autofinanciado & $\begin{array}{l}\text { Educación a } \\
\text { distancia }\end{array}$ \\
\hline
\end{tabular}

Fuente: Valdiviezo, 1999.

Es importante indicar que el proyecto Nuestros Niños Nuestro futuro empezó a funcionar gracias al convenio realizado entre la Facultad de Educación y la Fundación High Scope de Michigan, contando con el apoyo financiero de AID Washington y con las instituciones Fasinarm de Ecuador y Caritas de Bolivia. Este proyecto inició una nueva era en el desarrollo de proyectos sociales en la Facultad, siendo una experiencia piloto de capacitación e investigación-acción en la que el público objetivo fue el protagonista principal en la toma de decisiones.

En el año 1992 se inició el Proyecto Calidad de la Educación y Desarrollo Regional en el marco de los convenios macro que realizó la universidad con el Ministerio 
de Educación, con el apoyo financiero de la Agencia Española de Cooperación Internacional y con la asistencia técnica de la Universidad Nacional de Educación a Distancia (UNED) de Madrid, España.

Los objetivos del proyecto estuvieron encaminados a la mejora académica y técnico-pedagógica de los docentes en ejercicio que laboraban en los distintos niveles educativos, así como a la consolidación del sistema de educación a distancia como estrategia válida para la especialización, capacitación y perfeccionamiento del magisterio nacional. El proyecto se desarrolló desde tres líneas de trabajo: la especialización docente a través del Diploma de Segunda Especialidad en Formación Magisterial, dirigido fundamentalmente a docentes de institutos superiores pedagógicos interesados en la formación continua; la capacitación y actualización a través del curso Cómo elaborar un Proyecto de Innovación Educativa y cursos afines dirigidos a docentes y a profesionales de otras áreas con experiencia en el campo educativo e interesados en la investigación y en la diversificación curricular; y la capacitación en educación a distancia mediante el desarrollo de seminariosy detalleres a cargo de expertos nacionales e internacionales en el tema. Se estableció una red de comunicación conformada por la Pontificia Universidad Católica del Perú como sede central y por seis centros de apoyo localizados en las ciudades de Arequipa, Chiclayo, Lima, Puno, Tarapoto y Urubamba. Se elaboró material educativo consistente en 29 unidades de aprendizaje, 13 guías de estudio y 13 casetes de audio.

\begin{tabular}{|c|c|c|c|c|c|}
\hline \multicolumn{6}{|c|}{ RADIO DE ACCIÓN DEL PROYECTO } \\
\hline $\begin{array}{l}\text { Centro de } \\
\text { Apoyo Lima }\end{array}$ & $\begin{array}{l}\text { Centro de } \\
\text { Apoyo Chi- } \\
\text { clayo }\end{array}$ & $\begin{array}{l}\text { Centro de } \\
\text { Apoyo Areq- } \\
\text { uipa }\end{array}$ & $\begin{array}{l}\text { Centro de } \\
\text { Apoyo Puno }\end{array}$ & $\begin{array}{l}\text { Centro } \\
\text { de Apoyo } \\
\text { Chiclayo } \\
\text { Tarapoto }\end{array}$ & $\begin{array}{l}\text { Centro } \\
\text { de Apoyo } \\
\text { Chiclayo } \\
\text { Urubamba }\end{array}$ \\
\hline $\begin{array}{l}\text { Lima } \\
\text { Ancash } \\
\text { Ica } \\
\text { Huanuco } \\
\text { Cerro Pasco } \\
\text { Junín } \\
\text { Ayacucho }\end{array}$ & $\begin{array}{l}\text { Tumbes } \\
\text { Piura } \\
\text { Lambayeque } \\
\text { La Libertad } \\
\text { Cajamarca } \\
\text { Amazonas }\end{array}$ & $\begin{array}{l}\text { Arequipa } \\
\text { Moquegua } \\
\text { Tacna }\end{array}$ & Puno & $\begin{array}{l}\text { San Martín } \\
\text { Loreto } \\
\text { Ucayali }\end{array}$ & $\begin{array}{l}\text { Cusco } \\
\text { Madre de } \\
\text { Dios } \\
\text { Apurimac }\end{array}$ \\
\hline
\end{tabular}

Cuadro: Radio de acción del proyecto (Tueros, 1999).

Durante los años 1997 y 1998 el Departamento de Educación participó en la realización de seminarios virtuales sobre temas de actualidad en educación, 
que concitó interés por la calidad de los profesionales participantes del país y del extranjero. Cada seminario tuvo una duración aproximada de dos meses, tiempo en el cual se empleó el sistema electrónico para favorecer la cobertura.

\section{La educación a distancia en la formación de pre-grado}

En 1997 después de un proceso de estudio exhaustivo del mercado laboral realizado con estudiantes y egresados, tomando los aportes de los seminarios nacionales de Análisis y Perspectivas de la Educación en el Perú realizados durante los años 1980, 1985, 1990 y 1995, las evaluaciones de impacto, las demandas a la Facultad, y como producto del consenso de profesores y autoridades se elaboró una reforma curricular con el objetivo de responder a las demandas de la época sobre formación docente.

La Reforma Curricular, actualmente vigente, está basada en “(...) una reflexión filosófica-antropológica que busca definir nuestra concepción del ser humano que subyace en nuestras intencionalidades educativas (...), una aproximación socio cultural en la que sustentamos algunos conceptos- claves sobre el sentido del currículo en nuestra sociedad. Finalmente, (...) nuestra perspectiva más específicamente educativa...de la manera cómo enfocamos el proceso educativo" (PUCP-Facultad de Educación, 1997, p. 29).

Presenta características específicas que responden a las demandas de la formación de formadores en nuestra sociedad. El currículo de la Reforma es entendido como un proceso abierto y dinámico que se va alimentando gracias a experiencias humanas significativas, las mismas que se traducen en un proyecto curricular con elementos articulados. Es a la vez un proceso y un proyecto; como proceso es un currículo en construcción que se va formando poco a poco a la luz de variables de la misma universidad como del entorno. Como proyecto recoge las intenciones del grupo humano y las traduce en un proyecto curricular con elementos coherentes y articulados que deben ser planificados, desarrollados y evaluados en forma permanente, asumidos desde la investigación-acción. (PUCP-Facultad de Educación, 1997).

Recogiendo los aportes de la Comisión presidida por Delors (2003), el currículo trata de construir con la persona su posición frente al entorno, su posibilidad de ubicación en la vida, de búsqueda de plenitud y de establecimiento de diálogo y comunicación con los otros. Además, persigue el reconocimiento del futuro 
profesional de la educación como agente de cambio en la creación de una cultura democrática.

Para viabilizar la propuesta, el Plan de Estudios se ha organizado en tres Fases Formativas, cada una de las cuales constituye una etapa del currículo que "articula el proceso de formación del estudiante” (PUCP-Facultad de Educación, 1997, p. 60) de acuerdo a tres criterios: tipo de atención al alumno, modalidad de aprendizaje, y tipo de contenidos. La primera fase abarca del primer al tercer ciclo, la segunda fase del cuarto al sexto ciclo y la tercera fase del séptimo al décimo ciclo.

A partir de la segunda fase la modalidad de aprendizaje es mixta: presencial y a distancia; siendo creciente el empleo de la educación a distancia a medida que el estudiante va acercándose a la culminación de su formación profesional.

Cada uno de los cursos a distancia cuenta con sesiones presenciales durante su realización; la frecuencia de las sesiones varía de acuerdo a la naturaleza de la asignatura; puede haber desde una sola sesión presencial al inicio del curso, hasta sesiones presenciales quincenales. Acompañando el proceso de aprendizaje en los cursos a distancia el estudiante cuenta tanto con un profesor del curso como con materiales de estudio organizados en módulos de aprendizaje. El profesor es quien orienta el trabajo retroalimentando periódicamente al alumno en su trabajo individual y los materiales de estudio concentran las actividades que debe realizar. Estos constan de guías de estudio y de selecciones de textos según la temática materia de aprendizaje. Los estudiantes deben realizar las actividades planteadas en las guías y elaborar productos como resultados del aprendizaje.

Como se ha manifestado, la Reforma Curricular vigente en la Facultad incorpora varios aspectos nuevos en la formación del futuro docente, y dentro de ellos el estudio a distancia como modalidad alternativa que descarga la presencialidad de las clases y permite que los estudiantes de las dos últimas fases del plan de estudios puedan desarrollar su autonomía en el aprendizaje y dedicarse a otras actividades propias de su formación profesional. Dentro de esta óptica, apoya la inserción laboral del estudiante al prepararlo para enfrentar sistemas de estudio no convencionales y posibilita la formación oportuna y pertinente de todos los docentes de la Facultad en el uso de las diferentes tecnologías de que dispone la universidad, así como en el diseño y ejecución de cada materia bajo el sistema de educación a distancia. 


\section{Planes Especiales de Bachillerato y Licenciatura}

En el año 1981 la Facultad consciente de la necesidad de mejorar la calidad de la docencia en los distintos niveles educativos escolares, comenzó a ofrecer un plan de estudios denominado Plan de Complementación Pedagógica conducente a la obtención del título de Licenciado en Educación para los Bachilleres egresados de la PUCP y de Facultades de Teología de reconocidas universidades.

Dada la continuidad de su servicio en el tiempo, desde el año 2000 el acceso al plan de Complementación se extendió a los bachilleres egresados de otras universidades y a docentes en ejercicio. Tiene como objetivo complementar la formación de profesionales provenientes de diversos campos o especialidades, que deseen prepararse para el ejercicio docente en los niveles de Educación Secundaria y de Educación Superior, con miras a obtener la Licenciatura en Educación.

El Plan de Estudios está organizado en las mismas áreas que el de pre-grado: Aprender a aprender, Aprender a educar, Aprender a ser y Aprender a convivir en comunidad y con el entorno. Se desarrolla en dos semestres académicos mediante la modalidad de educación mixta: presencial y a distancia. Los participantes emplean guías didácticas, textos de lectura y materiales complementarios en los diferentes cursos. La asesoría se realiza mediante el teléfono y el correo electrónico.

Características similares tiene el plan conducente al Bachillerato, dirigido a docentes egresados de Institutos Superiores Pedagógicos, Escuela Nacional de Música, Escuela Superior de Bellas Artes y Escuela Nacional de Folklore.

\section{De los proyectos de desarrollo a los diplomas de especialización diseñados en la modalidad de Educación a Distancia}

En concordancia con la política de contribuir al mejoramiento de la calidad educativa y al desarrollo social del país, la Facultad de Educación, desde 1994, ofrece Diplomas de Segunda Especialidad a docentes que laboran en distintos niveles educativos, sectores y zonas geográficas de nuestro país. La mayoría de los Diplomas tienen sus antecedentes en las experiencias realizadas como proyectos sociales y todos se encuentran enmarcados dentro del sistema de educación a distancia. 


\section{Diploma de Segunda Especialidad Currículo y Metodología en Educación Inicial}

Se originó en 1994 en el Proyecto Nuestros Niños Nuestro Futuro, cuyos inicios se encuentran descritos en apartados anteriores. El propósito del Diploma fue mejorar la calidad de los servicios educativos en los Centros de Educación Inicial y en los programas no escolarizados y capacitar a su personal empleando una estrategia a distancia que permitiera el acceso al personal en servicio tanto de Lima como del interior del país, aun de zonas alejadas a los centros de formación y de especialización. Con el diploma pudieron continuar su formación, docentes de los diferentes niveles educativos, bachilleres en educación, profesionales en carreras afines (psicólogos, trabajadores sociales), estudiantes del último año de Educación Inicial, y promotores de Centros de Educación Inicial. El contenido curricular del Diploma se desarrolló en 20 meses mediante la estrategia a distancia, contó con 30 módulos impresos, distribuidos en 5 entregas. Cada entrega comprendió 6 módulos impresos, que constituyeron el material básico y un casete de audio conteniendo dos programas como material complementario. El Diploma dejó de funcionar en el año 2001.

\section{Diploma de Segunda Especialidad en Atención integral al niño menor de tres años}

Este diploma nació en el año 2000 sobre la base de los proyectos Nuestros Niños Nuestro Futuro y Nuestros Niños y la Comunidad como respuesta a la falta de personal capacitado en la atención a los niños en sus tres primeros años de vida, desde un enfoque integral. Se sabe que esta etapa en la vida del niño es crucial en el desarrollo de su personalidad y también en la manera de afrontar posteriormente las influencias de su entorno, razones más que suficientes para que docentes y encargados respondan a estas necesidades previniendo, así, futuros problemas de aprendizaje.

El Diploma tiene como objetivo contribuir en la formación profesional de los participantes desarrollando sus habilidades y actitudes que les permitan ofrecer un servicio educativo integral y de calidad dirigido a niños y niñas menores de tres años. Además, busca brindar las bases científicas que les permitan reconocer la importancia de la estimulación temprana para el desarrollo de los niños de esta edad, promover el desarrollo de sus habilidades para la organización e implementación de servicios de atención infantil, formales o no formales, con participación activa de los padres de familia; desarrollar estrategias metodológicas pertinentes a los niños y las niñas menores de tres años, articulando la programación curricular pertinente; y fomentar la prevención en relación al cuidado de la salud física y mental de los niños y las 
niñas en el aula, con la participación de los padres de familia y de la comunidad Está dirigido a profesionales que se desempeñan en programas de atención a niños y niñas menores de tres años, a profesionales en general interesados en el tema y a promotores de estos.

El Diploma se desarrolla en tres trimestres académicos bajo la modalidad de educación a distancia, modalidad en la que se da prioridad "(...) al estudio independiente de los participantes, capacitándolos para aprender a aprender y aprender a hacer de forma flexible, con autonomía en cuanto al uso del tiempo, estilo, ritmo y método de aprendizaje" (Vargas, J. 2002, p. 16).

\section{Diploma de Segunda Especialidad en Formación Magisterial}

El Diploma de Segunda Especialidad en Formación Magisterial se inició en 1994 como parte del proyecto Calidad de la Educación y Desarrollo Regional. Proyecto que surgió del III Seminario Análisis y Perspectivas de la Educación en el Perú: Educación para la Democracia en Participación Responsable, realizado en octubre de 1989 por la Facultad de Educación. En el marco del convenio que celebraron la Pontificia Universidad Católica del Perú, el Ministerio de Educación y la Agencia Española de Cooperación Internacional (AECI), se acordó atender la mejora de la calidad de la educación y este reto fue asumido por el Centro de Investigaciones y Servicios Educativos de la Facultad (CISE/PUCP).

El Diploma también recogió los resultados de la aplicación del Proyecto Profesionalización docente en zonas rurales andinas del Perú, realizado en 1988 por la Facultad con la participación del Instituto Superior Tecnológico y Pedagógico de Urubamba (Cusco), como centro piloto; proyecto que hemos mencionado en el apartado correspondiente.

Fue creado con el objetivo de "aportar al mejoramiento del desempeño profesional del formador de futuros educadores que le permita elevar la calidad educativa de las instituciones de nivel superior y contribuir en los procesos de descentralización asumiendo un compromiso ético, social y político" (Escobar y otros, 2001. p. 8). Además, busca orientar la reflexión del formador de formadores sobre la transcendencia de la educación así como sobre la realidad peruana actual, su quehacer educativo y su compromiso social frente a las exigencias actuales en el marco de una educación en valores y de la ética profesional docente; proporciona los lineamientos necesarios para planificar institucional y curricularmente de acuerdo a requerimientos regionales y nacionales, y brinda las bases teóricas para el análisis y aplicación de los elementos integrantes de un modelo didáctico de educación superior. 
El Diploma se desarrolla desde tres ejes: desarrollo profesional, investigación e innovación educativa y ética profesional e identidad cultural, que se cumplen desde cursos dictados en tres semestres académicos bajo la modalidad de educación a distancia con dos sesiones presenciales. El material educativo está conformado por unidades didácticas, por una guía del participante, y una página web. Esta última planificada especialmente con dos áreas: una abierta a cualquier visitante con el objeto de brindar información sobre el Diploma, y la otra restringida a los usuarios del mismo.

Asimismo, los participantes cuentan con distintas herramientas y ámbitos para el aprendizaje, tales como: correo electrónico, listas de distribución, foros, calendarioagenda y una sala de exposición virtual.

\section{Diploma de Segunda Especialidad en Educación Intercultural}

El Diploma se enmarcó dentro de las propuestas que se plantean en América Latina y Europa sobre interculturalidad, como respuesta a la valoración de la diversidad cultural y de la necesidad de aprender a vivir entre lo diverso. Además, para su implantación se consideró el acumulado de la Facultad de Educación en materia de Diplomas de Segunda Especialidad, los mismos que han incorporado el tema intercultural como eje transversal; también se tuvo en cuenta la elaboración de los lineamientos del Proyecto de formación de especialistas en Educación Bilingüe Intercultural en América Latina (Sub Región Andina), que fue presentado a la GTZ en 1997. Este Diploma contribuyó a optimizar la competencia profesional de educadores y demás profesionales que trabajan con grupos culturales diversos. Además, orientó la reflexión sobre la realidad pluricultural y multilingüe actual, el quehacer educativo y el compromiso social en el marco de una educación intercultural; actualizó los conocimientos de los participantes sobre la realidad peruana vista interdisciplinarmente con el propósito de comprenderla a cabalidad; y promovió el diseño y evaluación de proyectos de desarrollo educativos alternativos desde una mirada intercultural. El plan de estudios se desarrolló desde tres ejes rectores: interculturalidad, interdisciplinariedad e investigación, y dos áreas de estudio: el área de desarrollo personal- y el área de desarrollo técnico - pedagógico. El Diploma utilizó la combinación de dos modalidades educativas: presencial y a distancia, realizándose una interacción directa entre los docentes y los participantes, así como entre los participantes entre sí en la etapa presencial; y dando énfasis al estudio independiente a través de materiales educativos auto-instructivos y de la asesoría de profesores desde diversos medios de comunicación, en la etapa a distancia. Actualmente este Diploma se encuentra en fase de reestructuración. 


\section{Diploma de Segunda Especialidad en Proyectos Educativos y Cultura de Paz}

Se inició en 1998 como parte de la experiencia de los proyectos de formación docente: Educación y Cultura de Paz y Hacia una Escuela de Calidad que el Centro de Investigaciones y Servicios Educativos empezó en 1989. Buscó la promoción de actitudes favorables de los docentes en ejercicio hacia una Cultura de Paz en la comunidad educativa, proporcionándoles estrategias prácticas innovadoras que permitieran el diseño de proyectos educativos orientados al desarrollo del autoconocimiento, la autonomía, la responsabilidad y la clarificación y organización de un sistema de valores en los alumnos.

El público objetivo fue el personal directivo, los maestros en ejercicio (bachilleres, licenciados o titulados en educación), y otros profesionales interesados en promover una cultura de paz desde sus instituciones. En su desarrollo se empleó una modalidad mixta que combinaba la modalidad presencial y la modalidad a distancia. Durante el estudio a distancia, los participantes estudiaron con ayuda de módulos antológicos y de guías didácticas. En la etapa presencial se trabajó con una metodología activo - participativa. Actualmente se encuentra en proceso de reestructuración.

\section{Diploma de Segunda Especialidad Cultura Juvenil y Desarrollo}

En el año 2003 se inició el Diploma de Segunda Especialidad Cultura Juvenil y Desarrollo con el objeto de especializar a profesionales de diversas áreas en el trabajo con adolescentes y jóvenes, a fin de promover el conocimiento de políticas sociales y metodológicas de actuación con jóvenes. Además, este Diploma buscaba que los participantes profundizaran en el conocimiento teórico y científico sobre la juventud y en el análisis de distintos escenarios en los que se desenvuelven los jóvenes de nuestro país en el siglo XXI; desarrollaran estudios e investigaciones significativas sobre el fenómeno juvenil, y diseñaran y ejecutaran propuestas creativas para anticiparse y atender las demandas juveniles, tanto desde el estado como desde la sociedad civil, en coordinación con programas existentes, desde un enfoque participativo y comprometido con una sociedad justa, fraterna y solidaria. Estuvo dirigido a profesionales titulados de universidades e institutos superiores que en ese momento se encontraban trabajando en temas o proyectos vinculados a la juventud, o que estuvieran interesados en ellos. El Diploma estuvo organizado en dos áreas curriculares: el área de estudios interdisciplinares y el área de gestión de proyectos. Se desarrolló en tres ciclos académicos bajo la modalidad de educación mixta: a distancia con tres sesiones presenciales en cada curso; con material educativo 
organizado en módulos antológicos y complementado con guías de estudio. Los participantes hicieron uso de herramientas para el aprendizaje virtual, tales como correo electrónico, foros, calendario-agenda, y chats. Actualmente el Diploma se encuentra en etapa de reestructuración.

\section{Diploma de Segunda Especialidad en Enseñanza de Inglés como Lengua Extranjera}

Con el objeto de mejorar la competencia profesional de los docentes que se desempeñan como profesores de inglés sin que esa sea su especialidad de formación, y de esa manera contribuir al mejoramiento de la calidad de la enseñanza del idioma inglés a nivel nacional, favoreciendo la investigación en el área específica de la enseñanza de idiomas, en el año 2004 se inició el Diploma de Segunda Especialidad en Enseñanza de Inglés como Lengua Extranjera. Este Diploma se ofrece en dos semestres académicos, desde dos áreas de estudio: el área didáctica y el área de lingüística. El plan de estudios se desarrolla en modalidad mixta; la primera etapa de estudios es presencial, en la cual se cumplen tres días de orientación para realizar actividades diversas como tomar conocimiento del curso, distribuir los materiales, explicar las modalidades y presentar el cronograma de trabajo. Además se desarrollan dos cursos en modalidad mixta, uno en modalidad presencial, y cinco en modalidad a distancia virtual, de acuerdo a la naturaleza y a los objetivos de los mismos. Los materiales de lectura y guías didácticas facilitan la comprensión y aplicación de los temas desarrollados.

\section{Diploma de Segunda Especialidad Didáctica de la Matemática en Educación Primaria}

El Diploma se inició en el presente año como una respuesta a la necesidad de mejorar los logros de los estudiantes de educación básica en el aprendizaje de la Matemática; así como a la urgencia de definir las capacidades del pensamiento lógicomatemático que deben desarrollar los niños y niñas para enfrentar los desafíos del siglo XXI. Tiene como objetivo principal fortalecer las competencias de los docentes de educación primaria en la didáctica aplicada al área lógico-matemática. El diploma se desarrolla en dos ciclos académicos y consta de siete módulos de aprendizaje que se plantean como ejes temáticos, seleccionados por su importancia en la Matemática para la Educación Primaria. Está diseñado en la modalidad de educación mixta: a distancia y con tres encuentros presenciales (al inicio, a la mitad y final del diploma) 
de dos días de duración cada uno y de asistencia obligatoria. Los participantes emplean materiales impresos, así como herramientas de comunicación e información dispuestas en el Campus Virtual de la PUCP.

Diploma de Segunda Especialidad Gestión de Instituciones Educativas Promotoras de la Salud

El Diploma nació el presente año como una respuesta a la necesidad de contar con personas capacitadas para implementar servicios y entornos saludables que permitan la promoción de la salud integral desde la escuela. Tiene como objetivo formar recursos humanos para implementar instituciones educativas que promuevan la salud teniendo en cuenta la realidad local, regional o nacional. Está dirigido a profesionales en educación que laboran en el magisterio nacional, a especialistas en educación y a las personas responsables de la promoción de salud. Consta de tres ciclos de estudio desarrollados bajo la modalidad de educación a distancia virtual, empleando la plataforma virtual de la PUCP, material multimedia (CD) y material impreso.

Diploma de Segunda Especialidad en Gestión y Didáctica de Programas de Educación a Distancia

En el presente año la Facultad de Educación con el apoyo de la Facultad de Ciencias e Ingeniería y el auspicio de la Red Global para el Aprendizaje (GDLN) ofrece "una especialización en la gestión y didáctica de programas que se desarrollan en la modalidad de educación a distancia con énfasis en la integración de las tecnologías de información y comunicación (TIC)" (Patiño, 2004, p. 3). El Diploma tiene como objetivo formar especialistas en la gestión de programas de educación a distancia y en el diseño de entornos de aprendizaje, incorporando estrategias de las nuevas tecnologías de la información y de la comunicación.

Está diseñado en la modalidad de educación a distancia, combinando la metodología de la enseñanza virtual con el empleo de materiales multimedia y el uso de herramientas de aprendizaje colocadas en el campus virtual de la universidad. Se realizan diversas actividades en línea, tales como: foros de discusión, sesiones de chat, evaluaciones en línea, autoevaluaciones, análisis de lecturas. 


\section{Diploma de Segunda Especialidad en Políticas Educativas y Desarrollo Regional}

Este Diploma surge en el marco del proyecto Políticas Educativas y Desarrollo Regional que organiza la Facultad de Educación. Busca formar a profesionales con las competencias necesarias que les permitan participar en las instancias decisivas del ámbito político-educativo, reflexionando, investigando, evaluando y generando políticas educativas. Asimismo, pretende propiciar el intercambio de experiencias entre profesionales latinoamericanos en relación a la problemática, tendencias y alternativas del desarrollo de la educación y al rol de las políticas educativas dentro de este proceso. El Diploma tiene como público objetivo a los profesionales de la educación y de áreas afines que se encuentran interesados en el diseño, desarrollo y evaluación de políticas educativas a nivel regional. Se desarrolla en tres semestres académicos, bajo una modalidad mixta: a distancia con sesiones presenciales. El periodo a distancia se realiza mediante el empleo de la plataforma virtual de la PUCP.

\section{NUEVA OFERTA EN EDUCACIÓN A DISTANCIA}

Con una mirada prospectiva, la Facultad de Educación viene ampliando su oferta académica en educación continua mediante la puesta en marcha de Diplomas de Segunda Especialidad en temáticas diversas que tienen como propósito atender las demandas emergentes en materia de educación, desarrolladas en la modalidad de educación a distancia. A continuación dos ejemplos de esta oferta:

\section{Diploma de Segunda Especialidad en Educación Inclusiva y Atención a la Diversidad}

El Diploma de Educación Inclusiva y Atención a la Diversidad surge frente a la necesidad cada vez más creciente de brindar una respuesta educativa de calidad y con equidad a la diversidad, desde el desarrollo profesional de los docentes tanto en su formación inicial como continua. Se orienta a formar un especialista reflexivo y crítico del quehacer educativo capaz de asumir con eficiencia y eficacia los desafíos que implican el desarrollo de espacios educativos para todos. Se desarrolla en tres ciclos académicos, organizados en módulos de estudio, bajo la modalidad de educación a distancia. Se emplean herramientas virtuales como foros de discusión, análisis de materiales de discusión y lecturas, sesiones de conversación y evaluaciones en línea. Estará abierto al público en el mes de Febrero de 2007. 


\section{Diploma de Segunda Especialidad: Enseñanza del Idioma Español a Ha- blantes de otras Lenguas}

El Diploma de Segunda Especialidad Enseñanza del Idioma Español a Hablantes de otras Lenguas es un programa diseñado en la modalidad de educación a distancia que ofrece formación en metodología para la enseñanza de la lengua española en el marco de la cultura peruana. Se basa en los principios de la enseñanza comunicativa de las lenguas y constituye una interesante alternativa para quienes desean iniciarse en la didáctica de esta lengua, y para quienes están interesados en capacitarse en la metodología de segundas lenguas. Tiene como objetivo capacitar en la enseñanza del idioma español a quienes ya lo dominan, sobre la base de la formación académica que poseen. El Diploma está planteado desde tres áreas de estudio: el área sociolingüística, el área pedagógica, y el área psicológica, desarrolladas en dos modalidades de acuerdo a las necesidades y preferencias de los participantes: modalidad semi-presencial para los alumnos de Lima así como para quienes les sea más cómodo asistir a las sesiones presenciales, y modalidad a distancia para los participantes de provincias y del extranjero. Ambas modalidades cuentan con el soporte de la plataforma virtual Intranet. El Diploma se ofrecerá al público en Noviembre del año 2007.

\section{APORTES DE LA FACULTAD DE EDUCACIÓN A LA MODALIDAD DE EDUCACIÓN A DISTANCIA}

Desde los inicios de la educación a distancia en la Facultad de Educación de la PUCP se han ido incorporando nuevos elementos que han permitido que esta modalidad experimente un crecimiento seguro sobre bases sólidas. Dichos elementos constituyen indicadores del aporte que nuestra Unidad ofrece a esta modalidad alternativa de estudio en coherencia con el objetivo estratégico de la PUCP, que considera la educación a distancia como un instrumento para "extender el impacto de la Universidad en la sociedad llevando nuestro modelo de formación integral hasta el lugar de residencia de todas aquellas personas que lo requieran" (Plan Estratégico Institucional 2000-2010, p. 6). Los aportes más significativos son:

- La respuesta innovadora a las necesidades educativas del país aprovechando la tecnología y extendiendo los servicios a mayor cantidad de usuarios.

- La reflexión teórica constante sobre los procesos y las estrategias pedagógicas a emplear en la modalidad y la evaluación operativa de los mismos.

- La incorporación de los resultados de investigaciones en los niveles académico y de gestión. 
- La producción de materiales educativos que responden a las necesidades de aprendizaje características de esta modalidad y que vinculan de una forma eficaz, la relación educativa convencional entre el docente y el alumno.

- La existencia de mecanismos eficientes de optimización de los procesos de producción y distribución de materiales, de tutorías y evaluación del aprendizaje.

- El desarrollo de un modelo de educación que combina el sistema tradicional de educación con el empleo de nuevas tecnologías de la información y de la comunicación, en un constante afán de humanizar las tecnologías.

- La formación de docentes de los diferentes niveles y especialidades educativas, en temáticas diversas relacionadas con aspectos conceptuales, procedimentales, actitudinales y axiológicos.

- La atención a poblaciones de diferentes zonas urbanas, urbano marginales, rurales, costeñas y de la sierra del país en sus necesidades de formación continua.

- La incorporación formal de la educación a distancia en la formación de pregrado como una manera de apoyar al estudiante en su inserción al mercado laboral.

- El contar con equipos de profesores especializados en el desarrollo de programas en el sistema de educación a distancia.

- El trabajo interdisciplinar con expertos de distintas especialidades en la elaboración de los programas.

- El tener como soporte de los programas de educación a distancia, los tres ejes transversales que sustentan al currículo de la Facultad de Educación: Ética y valores, Interculturalidad y Paz y Formación Ciudadana.

- La consolidación de la red tutorial, formada por profesionales capacitados para asesorar y orientar a los usuarios en las exigencias de esta modalidad.

- El mayor uso de la tecnología gracias a la plataforma virtual como apoyo al proceso de aprendizaje, que permite diseñar y desarrollar entornos para gestionar, administrar y evaluar las actividades educativas.

- El empleo de diferentes opciones de interacción y de retroalimentación, tales como foros de debate, sesiones de chat, etc. que posibilitan la interacción.

- El desarrollo de una cultura de aprendizaje por la que cada participante asume la responsabilidad de su propio aprendizaje en forma autónoma.

\section{COMENTARIO FINAL}

La experiencia acumulada en la Facultad de Educación en relación a la educación a distancia nos demuestra que esta modalidad permite brindar una formación de 
calidad dado su carácter flexible. Si bien la forma mixta de atención en la que se combina la presencialidad con el trabajo independiente permite una mayor libertad al alumno en la gestión de su espacio y de su tiempo dedicado al estudio, la educación a distancia basada en nuevas tecnologías introduce importantes cambios que posibilitan una mejor interacción entre los distintos agentes usuarios de esta modalidad. Ya sea que se emplee la modalidad mixta o la de distancia virtual, es necesario fortalecer un conjunto de elementos que aseguren una educación de calidad; tales como: la comunicación fluida, el empleo pertinente y organizado de distintos recursos para el aprendizaje; el desarrollo del aprendizaje autónomo; la formación de grupos de inter-aprendizaje; la calidad del material educativo y la tutoría como componente principal dentro del conjunto de servicios de apoyo al participante.

\section{REFERENCIAS BIBLIOGRÁFICAS}

Capella, J. (1999). Los fundamentos teóricos y la conceptualización de educación a distancia. En: Consorcio de Universidades. Educación a distancia y nuevas tecnologías. Lima: Southern Perú.

Coloma, C. (2000). Políticas y estrategias sobre formación docente formuladas y desarrolladas en la Facultad. En $V$ Seminario de Análisis y Perspectivas de la Educación en el Perú. Lima: Departamento de Educación, Pontificia Universidad Católica del Perú, 56-87.

Consorcio de Universidades (1999). Educación a distancia y nuevas tecnologías, Primera Reunión de Consulta Interinstitucional sobre Educación a distancia Postsecundaria y Universitaria. Lima: Southern Perú.

Delors, J. (2003). La Educación encierra un Tesoro. Informe a la UNESCO de la Comisión Internacional sobre la Educación para el Siglo XXI. Madrid: Santillana, UNESCO.

Díaz C. y Suárez G. (2001). Evaluación de impacto del Diploma de Segunda Especialidad Proyectos Educativos y Cultura de Paz. Educación, X (19), 93110.
Escobar, P. y otros. (2001). Propuesta del Diploma de Segunda Especialidad Formación Magisterial. Lima: Facultad de Educación -PUCP.

Escotet, M. A. (1980). Tendencias de la educación superior a distancia. San José, Costa Rica: Universidad Estatal a Distancia.

Facultad de Educación (1998). Diploma de Segunda Especialidad en Educación Intercultural. Lima: Facultad de Educación- PUCP.

Facultad de Educación (2004). Diploma de Segunda Especialidad Gestión y Didáctica de Programas en la Educación a Distancia. Lima: Facultad de Educación- PUCP.

Facultad de Educación (2005). Proyecto del Diploma de Segunda Especialidad Gestión de Instituciones Educativas Promotoras de la Salud. Lima: Facultad de Educación- PUCP.

Facultad de Educación (2006). Diploma de Segunda Especialidad Didáctica de la Matemática en Educación Primaria. Lima: Facultad de Educación- PUCP.

Facultad de Educación (2006). Diploma de Segunda Especialidad Educación Inclusiva y Atención a la Diversidad. 
Lima: Facultad de Educación- PUCP.

Ferrazzi, G. (2001). Lineamientos de Desarrollo del Diploma de Segunda Especialidad Proyectos Educativos y Cultura De Paz. Informe presentado a la Facultad de Educación para el estudio de sistematización de los Diplomas de Segunda Especialidad. Lima: Facultad de Educación - PUCP.

García Aretio, L. (coord) (1999). La tutoría en la UNED. Bases y orientaciones. Madrid: UNED.

García Llamas, J. L. (1990). El aprendizaje adulto en un sistema abierto y a distancia. Madrid: Narcea.

García Ponce, I. (2003). Diploma de Segunda Especialidad en enseñanza de Inglés como lengua extranjera. Lima: Facultad de Educación - PUCP.

García Ponce, I. (2006). Proyecto de Diploma de Segunda Especialidad: Enseñanza del idioma español a hablantes de otras lenguas. Lima: Facultad de EducaciónPUCP.

Lamas, P. (1999). Comentario a la ponencia Fundamentos teóricos y conceptualización de educación a distancia, en Consorcio de Universidades. Educación a distancia y nuevas tecnologías. Lima: SouthernPerú.

Patiño, A. (2003). Educación a distancia, una ya larga experiencia en área de educación de la Pontificia Universidad Católica del Perú. Revista Sinopsis de la PUCP, 46.

Perraton, H. (1993). The Costs, en Perraton, H. (ed.) Distance Education for Teacher Training. London: Routledge.

PNUD (2005). Informe sobre Desarrollo Humano, Perú 2005. Hagamos de la competitividad una oportunidad para todos. Lima.

Pontificia Universidad Católica del Perú (1997). Propuesta de Reforma Curricular delafacultad deEducación dela Pontificia Universidad Católica del Perú. Lima: Facultad de Educación (Documento de Trabajo).

Pontificia Universidad Católica del Perú (2000). Planeamiento Estratégico 200o2010. Lima: Facultad de EducaciónPUCP.

Tafur, R. y otros (2003). Diploma de Segunda EspecialidadCulturaJuvenilyDesarrollo. Lima: Facultad de Educación- PUCP.

Tueros, E. (1999). Experiencias, en Consorcio de Universidades Educación a Distancia y Nuevas Tecnologías, Primera Reunión de Consulta Interinstitucional sobre Educación a distancia Postsecundaria y Universitaria. Lima: Southern Perú.

UNESCO (2002). Proyecto regional de educación para América Latina y el Caribe. Documento de la primera Reunión Intergubernamental del Proyecto Regional de Educación para América Latina y el Caribe. La Habana, Cuba.

Valdiviezo, E. (1999). Experiencias, en Consorcio de Universidades: Educación a Distancia y Nuevas Tecnologías, Primera Reunión de Consulta Interinstitucional sobre Educación a distancia Postsecundaria y Universitaria. Lima: Southern Perú.

Vargas, J. (2002). Guía del Participante. Diploma de Segunda Especialidad en Atención al Niño Menor de Tres Años. Lima: Facultad de Educación PUCP. 


\section{PERFIL ACADÈMICO Y PROFESIONAL DE LA AUTORA}

Dra. Rosa María Tafur Puente, Doctora en Educación por la UNED (España). Docente del Departamento de Educación de la Pontificia Universidad Católica del Perú, coordinadora de la especialidad de Educación Secundaria. Coordinadora del Programa de Orientación y Tutoría Personalizada a los Estudiantes de la Facultad de Educación. Especialista en Problemas de Aprendizaje por la Universidad Nacional Mayor de San Marcos. Licenciada en Educación por la Pontificia Universidad Católica del Perú. Con especialización en Evaluación Institucional; con experiencia en Capacitación Docente, y en Auditoria Pedagógica.

E-mail: rtafur@pucp.edu.pe

DIRECCIÓN DE LA AUTORA

Rosa Tafur Puente

Pontificia Universidad Católica del Perú

Av. Universitaria 1802, San Miguel, Lima, Perú.

Fecha de recepción del artículo: 22/12/06

Fecha de aceptación del artículo: 27/02/07 